\title{
Identifying Language Features Associated with Needs of Ovarian Cancer Patients and Caregivers Using Social Media
}

Young Ji Lee ${ }^{1,2}$, Hyeju Jang ${ }^{5}$, Grace Campbell1,3, Giuseppe Carenini ${ }^{5}$, Teresa Thomas ${ }^{1}$, Heidi Donovan ${ }^{1,4}$

${ }^{1}$ School of Nursing, University of Pittsburgh; ${ }^{2}$ Department of Biomedical Informatics, School of Nursing, University of Pittsburgh;

${ }^{3}$ Department of Obstetrics, Gynecology, and Reproductive Sciences; ${ }^{5}$ Department of Computer Science, University of British Columbia

\section{Background:}

Social media has gained an attention as a source to learn the perspectives, values, and needs of patients and caregivers in naturalistic settings

- Thorough understanding of their concerns and needs is the first step to develop interventions for the target population

- Language written in the social media can be cues of needs, however, manually identifying those cues is time-consuming and labor-intensive

- We propose a text classification machine learning model using simple natural language processing techniques, to identify language features that are associated with needs of ovarian cancer (OvCa) patients and caregivers on social media data.

\section{Data Source:}

- Collected from the Cancer Survivors Network online peersupport forum (http://csn.cancer.org)

- Crawled postings between 1/2006 - 3/2016

1,947 users, 50,626 postings

- Collected information:

- User id, posting date, type of posting (initial / reply), posting

- Average number of posting: 26 (SD: 117.6, range: 1 1821)

- 757 users had only 1 posting

- Average online discussion board participation time (days): 405 (SD: 463.0, range: 0 3707)

\section{Method:}

- Only included initial postings of patients and caregivers $(n=855)$ to understand the reason they visited the online forum

- Two undergraduate students coded each posting with 12 types of needs based on the literature:

- Physical; psychological/emotional; family-related; social; interpersonal/intimacy; practical; daily living;

spiritual/existential; health information; patient-clinician communication; cognitive needs; miscellaneous (posting does not include any needs such as advertisement)

- Applied machine learning to build a computational model to decide whether a posting has the given need or not

- Used bag-of-words (BOW) features, which consider each word in a posting as a feature to classify needs

- Performed chi-square-based feature selection to automatically identify more important features for each need category

- Trained a model using the selected features, and evaluated the model using the F1 score, a performance metric for classification accuracy

\section{Results:}

- Classification agreement between two annotators (kappa)

- Average across needs: 0.73

Highest kappa: 0.93 (information needs)

- Lowest kappa: 0.5 (spiritual/existential needs)

- Top most frequently occurring needs across postings:

- Information needs $(n=456)$

- Social needs $(n=307)$

Psychological/emotional needs $(n=141)$

Physical needs $(n=109)$

- $38 \%$ of postings $(n=326)$ described multiple needs in the same posting

- Among postings with multiple needs, $40 \%$ of them $(n=130)$ described social and information needs together

- F1 score of each need classification (a measure of accuracy)

- Physical needs: 0.88

- Psychological needs: 0.84

- Social needs: 0.78

Health information needs: 0.81

\begin{tabular}{|l|c|c|}
\hline Need Type & n & k (kappa) \\
\hline Health system/information needs & 456 & 0.93 \\
\hline Social needs & 307 & 0.87 \\
\hline Psychological/emotional needs & 141 & 0.83 \\
\hline Physical needs & 109 & 0.88 \\
\hline Miscellaneous & 74 & 0.49 \\
\hline Family-related needs & 53 & 0.85 \\
\hline Practical needs & 35 & 0.87 \\
\hline Patient-clinician communication needs & 19 & 0.85 \\
\hline Interpersonal/intimacy needs & 14 & 0.42 \\
\hline Spiritual/existential needs & 10 & 0.5 \\
\hline Daily living needs & 5 & 0.57 \\
\hline Cognitive needs & 4 & 0.75 \\
\hline
\end{tabular}

Table 1. Need type statistics of data. $n$ : number of posts that contain the need type, $\mathrm{k}$ : Cohen's kappa value

\begin{tabular}{|l|c|}
\hline Need Type & $\mathbf{n}$ \\
\hline Information-social & 130 \\
\hline Information-physical & 65 \\
\hline Family-related, emotional need & 60 \\
\hline Information-emotional & 31 \\
\hline Physical-social & 30 \\
\hline
\end{tabular}

Table 2. A set of topics that are most frequently reported in the same posting

\begin{tabular}{|c|c|}
\hline Need Type & Important features for the classification \\
\hline Physical & $\begin{array}{l}\text { abdomen, abnormally, alleviate, back, } \\
\text { endoscopy, centimeter, colonoscopy, months }\end{array}$ \\
\hline $\begin{array}{l}\text { Psychological/ } \\
\text { emotional }\end{array}$ & $\begin{array}{l}\text { anger, anxiety, attack, beating, breathing, } \\
\text { coincidence, convince, cope }\end{array}$ \\
\hline Social & $\begin{array}{l}\text { access, action, admitted, advice, aggressive, } \\
\text { alone, anxious, angry, attention, avoid, battle }\end{array}$ \\
\hline $\begin{array}{l}\text { Health system/ } \\
\text { information }\end{array}$ & $\begin{array}{l}\text { access, advice, alleviate, appointment, } \\
\text { appreciate, attention, blood, behavior }\end{array}$ \\
\hline \multicolumn{2}{|c|}{$\begin{array}{l}\text { Table 3. Top influential words for for prediction of most frequently } \\
\text { reported need type }\end{array}$} \\
\hline \multicolumn{2}{|l|}{ Discussion: } \\
\hline \multicolumn{2}{|c|}{$\begin{array}{l}\text { The first study to develop an initial model for automatically } \\
\text { classifying needs of OvCa patients and caregivers on social } \\
\text { media data } \\
\text { - Result showed even using simple word features can detect } \\
\text { needs with high accuracy } \\
\text { - Same features can predict multiple needs (e.g., social and } \\
\text { information needs) which can be more important cues for } \\
\text { clinicians to understand patients } \\
\text { - Patients tend to write multiple needs in one posting, which } \\
\text { requires taking into account correlations between different needs } \\
\text { Suggests the potential of using multiple language features and } \\
\text { classification methods to develop more sophisticated model } \\
\text { Future work involves exploring other language features (e.g., } \\
\text { groups of words clustered by using topic modeling techniques, } \\
\text { taxonomies, etc.) }\end{array}$} \\
\hline
\end{tabular}

